\title{
Entropy Spectrum of a KS Black Hole in IR Modified Hořava-Lifshitz Gravity
}

\author{
Shiwei Zhou, ${ }^{1,2}$ Ge-Rui Chen, ${ }^{1}$ and Yong-Chang Huang ${ }^{1}$ \\ ${ }^{1}$ Institute of Theoretical Physics, Beijing University of Technology, Beijing 100124, China \\ ${ }^{2}$ Department of Foundation, Academy of Armored Forces Engineering, Beijing 100072, China \\ Correspondence should be addressed to Shiwei Zhou; zhousw783@163.com
}

Received 6 January 2014; Accepted 10 February 2014; Published 13 March 2014

Academic Editor: Xiaoxiong Zeng

Copyright (c) 2014 Shiwei Zhou et al. This is an open access article distributed under the Creative Commons Attribution License, which permits unrestricted use, distribution, and reproduction in any medium, provided the original work is properly cited. The publication of this article was funded by SCOAP . $^{3}$

\begin{abstract}
As a renormalizable theory of gravity, Hořava-Lifshitz gravity, might be an ultraviolet completion of general relativity and reduces to Einstein gravity with a nonvanishing cosmological constant in infrared. Kehagias and Sfetsos obtained a static spherically symmetric black hole solution called KS black hole in the IR modified Hořava-Lifshitz theory. In this paper, the entropy spectrum and area spectrum of a KS black hole are investigated based on the proposal of adiabatic invariant quantity. By calculating the action of producing a pair of particles near the horizon, it is obtained that the action of the system is exactly equivalent to the change of black hole entropy, which is an adiabatic invariant quantity. With the help of Bohr-Sommerfeld quantization rule, it is concluded that the entropy spectrum is discrete and equidistant spaced and the area spectrum is not equidistant spaced, which depends on the parameter of gravity theory. Some summary and discussion will be given in the last.
\end{abstract}

\section{Introduction}

In the 1970's, with the discovery of Hawking radiation and Bekenstein's proposal of black hole entropy, black hole thermodynamics has been built up successfully, which has opened a new field to study quantum theory and gravity theory [1-4]. Nowadays there has been some trouble on the statistic origin and quantization of black hole entropy for physicists on black hole thermodynamics. Since Bekenstein proposed that the horizon area of a nonextremal black hole is an adiabatic invariant classically and the horizon area of black hole is quantized in units of $l_{p}^{2}[5-8]$, there has been much attention paid to the quantization of black hole entropy spectrum and area spectrum. Hod proposed that if one employs Bohr's corresponding principle, the real part of the quasinormal mode frequency is responsible for the area spectrum of black hole $[9,10]$. Combining the proposal by Bekenstein for the adiabaticity of black hole horizon area and Hod's proposal, Kunstatter proved that entropy spectrum of a $d$-dimensional black hole is quantized and the result is in agreement with that of Hod and Bekenstein [11]. Later,
Maggiore gave a new interpretation of black hole quasinormal modes in connection to the quantization of black hole horizon area. An important statement in Maggiore's work is that the periodicity of a black hole in Euclidean time may be the origin of area quantization [12]. It is well known that for any background spacetime with a horizon in Kruskal coordinates, the period with respect to Euclidean time takes the form of $T=2 \pi / \kappa$, where $\kappa$ is the surface gravity of the horizon. Vagenas exclusively used the fact that the black hole horizon area is an adiabatic invariant quantum and derived an equally spaced entropy spectrum of a black hole with its value to be equal to that of Bekenstein [13]. Zeng et al. considered that the action $I$, action variable $I_{v}$, and Hamiltonian $H$ of any single periodic system satisfy the relation $I=I_{v}-\int H d t$. They proposed that the action variable can be quantized with the equally spaced form $I_{v}=2 \pi n \hbar$. Once the action and Hamiltonian are given, the quantization action variable can be obtained. With the help of Bohr-Sommerfeld quantization rule, they proved that the quantized action variable is nothing but the entropy of black hole; thus the entropy and the horizon area of a black hole 
can be quantized. They emphasized that the action variable should be adiabatic invariant [14-16]. Recently, Jiang and Han argued that the adiabatic invariant quantity $\int p_{i} d q_{i}$ is not canonically invariant, and the adiabatic invariant quantum should be of the covariance form $\oint p_{i} d q_{i}=n h$. They also obtained the equally spaced entropy spectrum with the form of $\Delta S=2 \pi$ [17]. Some more works on the quantization of entropy spectrum of a black hole can be seen in the paper [18-23].

Hořava gravity is a nonrelativistic renormalizable theory of gravitation [24], which is inspired by the anisotropic scaling between time and space in condensed matter systems in particular in the theory of quantum critical phenomena, where the degree of anisotropy between space and time is characterized by the "dynamical critical exponent" $z$. It is well known that relativistic systems automatically satisfy $z=1$ as a consequence of Lorentz invariance. In HořavaLifshitz theory, systems' scaling at a short distance exhibits a strong anisotropy between space and time with $z>1$. This will improve the short-distance behavior of the theory. The anisotropy at short distance can be lost for long distance while the Lorentz symmetry will appear as an emergent symmetry. The black hole solution of original Hořava-Lifshitz gravity does not recover the usual Schwarzschild-anti-de Sitter black hole with the detailed-balance condition. A relevant operator proportional to the $3 D$ geometry Ricci scalar of the original Hořava-Lifshitz theory action was introduced [25] and it deviated from detailed balance. This does not modify the ultraviolet properties of the theory. However, it modifies the infrared Hořava-Lifshitz gravity theory. So a Schwarzschildanti-de Sitter solution can be realized in infrared modified Hořava-Lifshitz gravity theory and the Minkowski vacuum is also allowed. On the limit of vanishing $\Lambda_{\omega}$, a spherically symmetric black hole solution has been obtained by Kehagias and Sfetsos [25], which is the analogy of Schwarzschild black hole in general relativity and is exactly asymptotically flat. After Hořava-Lifshitz gravity was proposed, much attention has been paid to it [26-31].

In this paper, based on Vagenas' proposal of adiabatic invariant quantity, we investigate entropy spectrum of a KS black hole in IR modified Hořava-Lifshitz gravity. Vagenas pointed that the general coordinates $q_{i}$ contain $q_{0}=\tau$ and $q_{1}=r$, and the contribution of the two parts to the adiabatic invariant quantity are equivalent with each other by defining $\dot{r}=d r / d \tau$. Thus the integration result of adiabatic invariant quantity was directly given by the $\tau$-integration, where the period $T$ of gravity system satisfies $T=2 \pi / \kappa$. We find that the periodicity of gravity system can conveniently be used to calculate the entropy spectrum. However, the physical picture of periodicity is not clear. We give our explanation about it by considering a process that a pair of particles create outside the horizon. One period of the system corresponds to the process with the outgoing positive energy particle crossing outwards the horizon while the negative energy particle tunnels into the black hole. The movement of the two particles can be described as a tunneling process proposed by Parikh and Wilczek's Hawking radiation [32]. After calculating, we find that the action of the system is exactly the black hole entropy which is the adiabatic invariant quantity. With the help of Bohr-Sommerfeld quantization rule, we obtain the quantized entropy and area spectrum. Some summary and discussion will be given in the latter.

\section{Review of a KS Black Hole in IR Modified Hořava-Lifshitz Gravity}

Using the ADM decomposition of the metric

$$
d s^{2}=-N^{2} d t^{2}+g_{i j}\left(d x^{i}+N^{i} d t\right)\left(d x^{j}+N^{j} d t\right),
$$

where $N$ and $N^{i}$ are the "lapse" and "shift" variables, respectively, and $g_{i j}$ is the spatial metric. On the limit of $\Lambda_{\omega} \rightarrow 0$, the action of IR modified Horrava-Lifshitz gravity theory can be given by

$$
\begin{aligned}
S=\int d t d^{3} x \sqrt{g} N \\
\times\left[\frac{2}{\kappa^{2}}\left(K_{i j} K^{i j}-\lambda K^{2}\right)\right. \\
\quad-\frac{\kappa^{2}}{2 \omega^{4}} C_{i j} C^{i j}+\frac{\kappa^{2} \mu}{2 \omega^{2}} \epsilon^{i j k} R_{i l}^{(3)} \nabla_{j} R_{k}^{(3) l} \\
\quad-\frac{\kappa^{2} \mu^{2}}{8} R_{i j}^{(3)} R^{(3) i j} \\
\left.\quad+\frac{\kappa^{2} \mu^{2}}{8(1-3 \lambda)} \frac{1-4 \lambda}{4}\left(R^{(3)}\right)^{2}+\mu^{4} R^{(3)}\right],
\end{aligned}
$$

which is obtained by introducing a term proportional to the Ricci scalar of the three-geometry $\mu^{4} R^{(3)}$ to the original action of Hořava-Lifshitz gravity [26, 27]. Here $K_{i j}$ is the extrinsic curvature, defined by

$$
K_{i j}=\frac{1}{2 N}\left(\dot{g}_{i j}-\nabla_{i} N_{j}-\nabla_{j} N_{i}\right)
$$

where the dot denotes a derivative with respect to $t, C^{i j}$ is the Cotton tensor defined by

$$
C^{i j}=\epsilon^{i k l} \nabla_{k}\left(R_{l}^{(3) j}-\frac{1}{4} R^{(3)} \delta_{l}^{j}\right),
$$

and $R^{(3)}$ is the 3-dimensional curvature scalar for $g_{i j}$; $\kappa, \lambda, \omega, \mu$ are all coupling constant parameters.

Comparing the action for the case of $\lambda=1$ with the standard Einstein-Hilbert action, we find that the Lagrangian will become the usual Einstein-Hilbert Lagrangian when the speed of light $c$, Newton's constant $G$, and the cosmological constant $\Lambda$ are given by

$$
c^{2}=\frac{\kappa^{2} \mu^{4}}{2}, \quad G=\frac{\kappa^{2}}{32 \pi c}, \quad \Lambda=\frac{3}{2} \Lambda_{W} .
$$

The spherically symmetric asymptotically flat black hole solution has been obtained by Cai et al. [27], which is the 
analogy of Schwarzschild black hole in general relativity. The metric can be written as

$$
d s^{2}=-f(r) d t^{2}+\frac{1}{f(r)} d r^{2}+r^{2}\left(d \theta^{2}+\sin ^{2} \theta d \varphi^{2}\right),
$$

with

$$
f(r)=1+\omega r^{2}-\sqrt{r\left(\omega^{2} r^{3}+4 \omega M\right)},
$$

where $M$ is an integration constant corresponding to the mass of black hole, and $\omega$ is a coupling constant parameter.

The condition $f\left(r_{ \pm}\right)=0$ gives the outer and inner horizons at

$$
r_{ \pm}=M\left(1 \pm \sqrt{1-\frac{1}{2 \omega M^{2}}}\right)
$$

To avoid naked singularity, we should have $\omega M^{2} \geq 1 / 2$. In the regime of traditional general relativity, we have $\omega M^{2} \gg$ 1 , so the outer horizon approaches the usual Schwarzschild horizon $r_{+} \simeq 2 M$, whereas the inner one approaches the singularity $r_{-} \simeq 0$.

\section{Entropy Quantization via Adiabatic Invariant Action}

We consider a process that a pair of particles create near the horizon. While the outgoing positive energy particle crossing outwards the horizon, the negative energy particle ingoing towards the black hole along the radial direction. We describe the movement of the two particles as a tunneling process proposed by Parikh and Wilczek's proposal [32].

The action of the system is

$$
\begin{aligned}
I & =\int p_{r} d r=\int_{r_{\text {in }}}^{r_{\text {out }}} p_{r} d r+\int_{r_{\text {out }}}^{r_{\text {in }}} p_{r} d r \\
& =\int_{r_{\text {in }}}^{r_{\text {out }}} \int_{0}^{p_{r}} d p_{r}^{\prime} d r+\int_{r_{\text {out }}}^{r_{\text {in }}} \int_{0}^{p_{r}} d p_{r}^{\prime} d r .
\end{aligned}
$$

The first term is corresponding to the particles with positive energy, and the second term is the negative energy one. When energy conservation is considered, the black hole mass will decrease with the outgoing particle emitting. The Hamilton $H$, ADM energy $M$, and the particle's energy $m^{\prime}$ satisfy the relation $H=M-m^{\prime}$; that is, $d H=-d m^{\prime}$. Then by use of Hamilton's equation $\dot{r}=d H / d p_{r}$, the first term of (9) becomes

$$
\begin{aligned}
I_{1} & \equiv \int_{r_{\text {in }}}^{r_{\text {out }}} \int_{0}^{p_{r}} d p_{r}^{\prime} d r=\int_{r_{\text {in }}}^{r_{\text {out }}} \int_{M}^{M-m} \frac{d H}{\dot{r}} d r \\
& =\int_{0}^{m} \int_{r_{\text {in }}}^{r_{\text {out }}} \frac{d r}{\dot{r}}\left(-d m^{\prime}\right),
\end{aligned}
$$

where $r_{\text {in }}=r_{h}(M)-\epsilon, r_{\text {out }}=r_{h}^{\prime}(M-m)+\epsilon$, for the reason of that the black hole horizon will decrease with the particle emitting out.
When considering $\dot{r}=d r / d \tau=f(r)$, we get

$$
I_{1}=\int_{0}^{m} \int_{r_{\text {in }}}^{r_{\text {out }}} \frac{d r}{1+\omega r^{2}-r\left[\omega^{2} r^{3}+4 \omega\left(M-m^{\prime}\right)\right]} d m^{\prime} .
$$

It is easily found that there is a pole at $r_{h}^{\prime}=\left(M-m^{\prime}\right)+$ $\sqrt{\left(M-m^{\prime}\right)^{2}-1 / 2 \omega}$. We do the integration as follows:

$$
\begin{array}{r}
I_{1}=\int_{0}^{m} \int_{r_{\text {in }}}^{r_{\text {out }}}\left(d r\left(r-r_{h}^{\prime}\right)\right. \\
\times[2 \omega r
\end{array}
$$

$$
\left.\left.\left.-\frac{2 \omega^{2} r^{3}+2 \omega\left(M-m^{\prime}\right)}{\sqrt{r\left(\omega^{2} r^{3}+4 \omega\left(M-m^{\prime}\right)\right)}}\right]\right)^{-1}\right) d m^{\prime}
$$$$
=2 \pi \int_{0}^{m}\left(1 \left(2 \omega r_{h}^{\prime}\right.\right.
$$

$$
\begin{aligned}
& \left.\left.-\frac{2 \omega^{2} r_{h}^{\prime 3}+2 \omega\left(M-m^{\prime}\right)}{\sqrt{r_{h}^{\prime}\left[\omega^{2} r_{h}^{\prime 3}+4 \omega\left(M-m^{\prime}\right)\right]}}\right)^{-1}\right) d m^{\prime} \\
& =2 \pi \int_{0}^{m}\left(\left(\frac{1}{2}+2 \omega\left(M-m^{\prime}\right)^{2}\right.\right. \\
& \left.+2 \omega\left(M-m^{\prime}\right) \sqrt{\left(M-m^{\prime}\right)^{2}-\frac{1}{2 \omega}}\right) \\
& \left.\times\left(2 \omega \sqrt{\left(M-m^{\prime}\right)^{2}-\frac{1}{2 \omega}}\right)^{-1}\right) d m^{\prime} \\
& =2 \pi\left[\int_{0}^{m} \frac{1}{4 \omega \sqrt{\left(M-m^{\prime}\right)^{2}-1 / 2 \omega}} d m^{\prime}\right. \\
& +\int_{0}^{m} \frac{\left(M-m^{\prime}\right)^{2}}{\sqrt{\left(M-m^{\prime}\right)^{2}-1 / 2 \omega}} d m^{\prime} \\
& \left.+\int_{0}^{m}\left(M-m^{\prime}\right) d m^{\prime}\right]
\end{aligned}
$$




$$
\begin{gathered}
=2 \pi\left[\frac{M}{2} \sqrt{M^{2}-\frac{1}{2 \omega}}-\frac{M-m^{\prime}}{2} \sqrt{\left(M-m^{\prime}\right)^{2}-\frac{1}{2 \omega}}\right. \\
+\frac{1}{2 \omega}\left[\ln M+\sqrt{M^{2}-\frac{1}{2 \omega}}-\ln (M-m)\right. \\
\left.\left.+\sqrt{(M-m)^{2}-\frac{1}{2 \omega}}\right]+M m-\frac{m^{2}}{2}\right] .
\end{gathered}
$$

The second term of (9) corresponds to ingoing particles with negative energy. After similar calculation as the first term, we find that the contribution is equivalent to that of $I_{1}$. That is,

$$
I=2 I_{1} \text {. }
$$

On the other hand, the Hawking temperature of the outer event horizon has been obtained as [31]

$$
\begin{aligned}
T_{H} & =\frac{2 \omega r_{+}^{2}-1}{8 \pi\left(\omega r_{+}^{3}+r_{+}\right)} \\
& =\frac{\sqrt{M^{2}-1 / 2 \omega}}{2 \pi\left(2 M^{2}+2 M \sqrt{M^{2}-1 / 2 \omega}+1 / 2 \omega\right)}
\end{aligned}
$$

which is proportional to the surface gravity $\kappa=$ $\left.(1 / 2)(\partial f(r) / \partial r)\right|_{r=r_{+}}$on the event horizon of black hole. Considering the first law of thermodynamics and substituting the expression of temperature of black hole, we can obtain the entropy

$$
S_{\mathrm{BH}}=\frac{A}{4}+\frac{\pi}{\omega} \ln \frac{A}{4}
$$

where $A=\pi r_{+}^{2}$ is the area of event horizon.

After calculation, we find that the change of black hole entropy $\Delta S$, when a particle with energy of $m$ emits out of the black hole, is exactly equivalent to the action $I$; that is,

$$
\Delta S=\pi\left[r_{+}^{2}(M)-r_{+}^{2}(M-m)\right]=I .
$$

Now, using the periodicity of the black hole, we calculate the adiabatic invariant quantity. According to the dimensional reduction technique, the two-dimensional spacetime of a KS black hole can be given by

$$
d s^{2}=-f(r) d t^{2}+f(r)^{-1} d r^{2}
$$

When defining the tortoise coordinate as

$$
r_{*}=r+\frac{1}{2 \kappa_{+}} \ln \frac{r-r_{+}}{r_{+}}+\frac{1}{2 \kappa_{-}} \ln \frac{r-r_{-}}{r_{-}},
$$

in which $\kappa_{ \pm}=\left.\left(f^{\prime}(r) / 2\right)\right|_{r=r_{ \pm}}=\left(2 \omega r_{ \pm}^{2}-1\right) /\left(4 r_{ \pm}\left(\omega r_{ \pm}^{2}+1\right)\right)$ is the surface gravity on the outer (inner) horizon. Using the null coordinates $u=t-r_{*}, v=t+r_{*}$, we can get the coordinates $U=-e^{-\kappa_{+} u}$ and $V=e^{\kappa_{+} v}[33,34]$. Then define

$$
\begin{aligned}
& T=\frac{1}{2}(V+U) \\
& =e^{\kappa_{+} r}\left(\frac{r-r_{+}}{r_{+}}\right)^{1 / 2}\left(\frac{r-r_{-}}{r_{-}}\right)^{\kappa_{+} / 2 \kappa_{-}} \sinh \kappa_{+} t, \\
& R=\frac{1}{2}(V-U)=e^{\kappa_{+} r}\left(\frac{r-r_{+}}{r_{+}}\right)^{1 / 2}\left(\frac{r-r_{-}}{r_{-}}\right)^{\kappa_{+} / 2 \kappa_{-}} \cosh \kappa_{+} t,
\end{aligned}
$$

where $T, R$ are the Kruskal-like coordinates.

Different from (18), that is,

$$
d r_{*}=f(r)^{-1} d r
$$

the two-dimensional KS metric becomes

$$
\begin{aligned}
d s^{2}= & -d t^{2}+d r_{*}^{2} \\
= & \kappa_{+}^{-2} e^{-2 \kappa_{+} r}\left(\frac{r-r_{-}}{r_{+}}\right)\left(\frac{r_{-}}{r-r_{-}}\right)^{\kappa_{+} / \kappa_{-}} \\
& \times\left[-d T^{2}+d R^{2}\right] .
\end{aligned}
$$

Transforming the time coordinate as $t \rightarrow-i \tau$, we have

$$
\begin{aligned}
& i T=e^{\kappa_{+} r}\left(\frac{r-r_{+}}{r_{+}}\right)^{1 / 2}\left(\frac{r-r_{-}}{r_{-}}\right)^{\kappa_{+} / 2 \kappa_{-}} \sin \kappa_{+} \tau, \\
& R=e^{\kappa_{+} r}\left(\frac{r-r_{+}}{r_{+}}\right)^{1 / 2}\left(\frac{r-r_{-}}{r_{-}}\right)^{\kappa_{+} / 2 \kappa_{-}} \cos \kappa_{+} \tau .
\end{aligned}
$$

It is easily found that both $T, R$ are periodic functions with respect to the Euclidean time $\tau$ with the period of $2 \pi / \kappa_{+}$. Since we only consider the case of outer horizon, for simplicity we write $\kappa_{+}$for $\kappa$ from now on.

When utilizing Vagenas's adiabatic invariant quantity with the following form:

$$
\begin{aligned}
I & =\int p_{i} d q_{i}=\iint_{0}^{p_{i}} d p_{i}^{\prime} d q_{i}=\iint_{0}^{H} \frac{d H^{\prime}}{\dot{q}_{i}} d q_{i} \\
& =\iint_{0}^{H} d H^{\prime} d \tau+\iint_{0}^{H} \frac{d H^{\prime}}{\dot{r}} d r,
\end{aligned}
$$

where $p_{i}$ are the conjugate momentum of the general coordinate $q_{i}$ with $i=0,1$ for $q_{0}=\tau$ and $q_{1}=r$. Considering $\dot{r}=d r / d \tau$, we have

$$
I=2 \iint_{0}^{H} d H^{\prime} d \tau
$$

Because of the periodicity of $\tau$ with $T=2 \pi / \hbar \kappa$, the adiabatic invariant quantity can be calculated as

$$
I=2 \pi \int_{0}^{H} \frac{d H^{\prime}}{\kappa}=\hbar \int_{0}^{H} \frac{d H^{\prime}}{T_{\mathrm{BH}}}=\hbar S_{\mathrm{BH}} \cdot
$$


Implementing the Bohr-Sommerfeld quantization condition

$$
\oint p d q=2 \pi n \hbar
$$

the black hole entropy spectrum can be given as

$$
S_{\mathrm{BH}}=2 \pi n, \quad n=1,2,3, \ldots,
$$

and the entropy spectrum is discrete and equidistant spaced with

$$
\Delta S_{\mathrm{BH}}=2 \pi
$$

To get the area spectrum, differentiate (15),

$$
\Delta S_{\mathrm{BH}}=\frac{1}{4} \Delta A+\frac{\pi}{\omega} \frac{4}{A} \Delta A,
$$

we have

$$
\Delta A=\frac{\Delta S_{\mathrm{BH}}}{1 / 4+4 \pi / A \omega} \simeq 8 \pi\left(1-\frac{4}{\omega r_{+}^{2}}\right) .
$$

We find that area spectrum is not equidistant spaced.

\section{Summary and Conclusion}

In this paper, based on the idea of adiabatic invariant quantity, we have investigated entropy spectrum of a KS black hole in IR modified Hořava-Lifshitz gravity. As a modified gravity theory, the entropy of a KS black hole does not satisfy Bekenstein's entropy-area relation. It consists of two terms: one is the Bekenstein-Hawking entropy, the other is a logarithmic term. The discrepancy between the entropy and the Bekenstein-Hawking entropy is the reflection of differences between this modified gravity theory and general relativity. After calculating, we find that the black hole entropy is an adiabatic invariant quantity. With the help of Bohr-Sommerfeld quantization rule, we obtain the quantized entropy and area spectrum. It is concluded that the entropy spectrum can be given as $S_{\mathrm{BH}}=2 \pi n$ with $n=1,2,3, \ldots$, which is discrete and equidistant spaced with $\Delta S_{\mathrm{BH}}=2 \pi$; and the area spectrum is not equidistant spaced, which depends on the parameter of gravity theory. In addition, by calculating the action of a production of a pair of particles near the horizon, we find that the action of the system is exactly equivalent to the change to black hole entropy, which is an adiabatic invariant quantity. The procession of the particle producing, with positive energy outgoing towards the horizon while the one with negative energy is ingoing the horizon, can give a clear explanation to the periodicity of gravity system.

\section{Conflict of Interests}

The authors declare that there is no conflict of interests regarding the publication of this paper.

\section{Acknowledgments}

This work is supported by Beijing Postdoctoral Research Foundation no. 71006015201201 and National Natural Science Foundation of China (no. 11275017 and no. 11173028).

\section{References}

[1] S. W. Hawking, "Particle creation by black holes," Communications in Mathematical Physics, vol. 43, no. 3, pp. 199-220, 1975.

[2] S. W. Hawking, "Black hole explosions?" Nature, vol. 248, no. 5443, pp. 30-31, 1974.

[3] S. W. Hawking, "Information loss in black holes," Physical Review D, vol. 72, no. 8, Article ID 084013, 4 pages, 2005.

[4] J. M. Bardeen, B. Carter, and S. W. Hawking, "The four laws of black hole mechanics," Communications in Mathematical Physics, vol. 31, pp. 161-170, 1973.

[5] J. D. Bebenstein, "Black holes and entropy," Physical Review D, vol. 7, no. 8, pp. 2333-2346, 1973.

[6] J. D. Bebenstein, "Black holes and the second law," Lettere Al Nuovo Cimento, vol. 4, pp. 737-740, 1972.

[7] J. D. Bebenstein, “The quantum mass spectrum of the Kerr black hole," Lettere Al Nuovo Cimento, vol. 11, pp. 467-470, 1974.

[8] J. D. Bebenstein, "Extraction of energy and charge from a black hole," Physical Review D, vol. 7, pp. 949-953, 1973.

[9] S. Hod, "Bohr's correspondence principle and the area spectrum of quantum black holes," Physical Review Letters, vol. 81, no. 20, pp. 4293-4296, 1998.

[10] S. Hod, "Gravitation, the quantum and Bohr's correspondence principle," General Relativity and Gravitation, vol. 31, no. 11, pp. 1639-1644, 1999.

[11] G. Kunstatter, " $d$-dimensional black hole entropy spectrum from quasinormal modes," Physical Review Letters, vol. 90, no. 16, Article ID 161301, 4 pages, 2003.

[12] M. Maggiore, "Physical interpretation of the spectrum of black hole quasinormal modes," Physical Review Letters, vol. 100, no. 14, Article ID 141301, 4 pages, 2008.

[13] B. R. Majhi and E. C. Vagenas, "Black hole spectroscopy via adiabatic invariance," Physics Letters B, vol. 701, pp. 623-625, 2011.

[14] X. X. Zeng, X. M. Liu, and W. B. Liu, "Periodicity and area spectrum of black holes," The European Physical Journal C, vol. 72, p. 1967, 2012.

[15] X. X. Zeng and W. B. Liu, "Spectroscopy of a ReissnerNordström black hole via an action variable," The European Physical Journal C, vol. 72, p. 1987, 2012.

[16] X.-M. Liu, X.-X. Zeng, and S.-W. Zhou, "Area spectra of BTZ black holes via periodicity," Science China Physics, Mechanics and Astronomy, vol. 56, no. 9, pp. 1627-1631, 2013.

[17] Q.-Q. Jiang and Y. Han, "On black hole spectroscopy via adiabatic invariance," http://arxiv.org/abs/1210.4002.

[18] H.-L. Li, R. Lin, and L.-Y. Chen, "Entropy quantization of Reissner-Nordström de Sitter black hole via adiabatic covariant action," General Relativity and Gravitation, vol. 45, pp. 865-875, 2013.

[19] D. Chen and H. Yang, "Entropy spectrum of a Kerr anti-de Sitter black hole," The European Physical Journal C, vol. 72, p. 2027, 2012.

[20] R. Tharanath and V. C. Kuriakose, "Thermodynamics and spectroscopy of charged dilaton black holes," General Relativity and Gravitation, vol. 45, no. 9, pp. 1761-1770, 2013. 
[21] Q. Q. Jiang, "Revisit emission spectrum and entropy quantum of the Reissner-Nordström black hole," The European Physical Journal C, vol. 72, p. 2086, 2012.

[22] C.-Z. Liu, "Black hole spectroscopy via adiabatic invariant in a quantum corrected spacetime," The European Physical Journal C, vol. 72, p. 2009, 2012.

[23] T. Tanaka and T. Tamaki, "Area spectrum of horizon and black hole entropy," The European Physical Journal C, vol. 73, p. 2314, 2013.

[24] P. Hořava, "Quantum gravity at a Lifshitz point," Physical Review D, vol. 79, no. 8, Article ID 084008, 15 pages, 2009.

[25] A. Kehagias and K. Sfetsos, "The black hole and FRW geometries of non-relativistic gravity," Physics Letters B, vol. 678, no. 1, pp. 123-126, 2009.

[26] H. Lü, J. Mei, and C. N. Pope, "Solutions to hořava gravity," Physical Review Letters, vol. 103, no. 9, Article ID 091301, 2009.

[27] R.-G. Cai, L.-M. Cao, and N. Ohta, “Topological black holes in Hořava-Lifshitz gravity," Physical Review D, vol. 80, no. 2, Article ID 024003, 7 pages, 2009.

[28] S. Mukohyama, "Scale-invariant cosmological perturbations from Hořava-Lifshitz gravity without inflation," Journal of Cosmology and Astroparticle Physics, vol. 2009, article 001, 2009.

[29] S. Mukohyama, K. Nakayama, F. Takahashi, and S. Yokoyama, "Phenomenological aspects of Horava-Lifshitz cosmology," Physics Letters B, vol. 679, no. 1, pp. 6-9, 2009.

[30] S.-W. Zhou and W.-B. Liu, "Three classical tests of HořavaLifshitz gravity theory," Astrophysics and Space Science, vol. 337, no. 2, pp. 779-784, 2012.

[31] S.-W. Zhou and W.-B. Liu, "Black hole thermodynamics of Hořava-Lifshitz and IR modified Hořava-Lifshitz gravity theory," International Journal of Theoretical Physics, vol. 50, no. 6, pp. 1776-1784, 2011.

[32] M. K. Parikh and F. Wilczek, "Hawking radiation as tunneling," Physical Review Letters, vol. 85, no. 24, pp. 5042-5045, 2000.

[33] C. Liang and B. Zhou, Differential Geometry and General Relativity, Science Press, Beijing, China, 2006.

[34] Z. Zhao, The Thermal Nature of Black Holes and the Singularity of the Space-Time, Beijing Normal University Press, Beijing, China, 1999. 

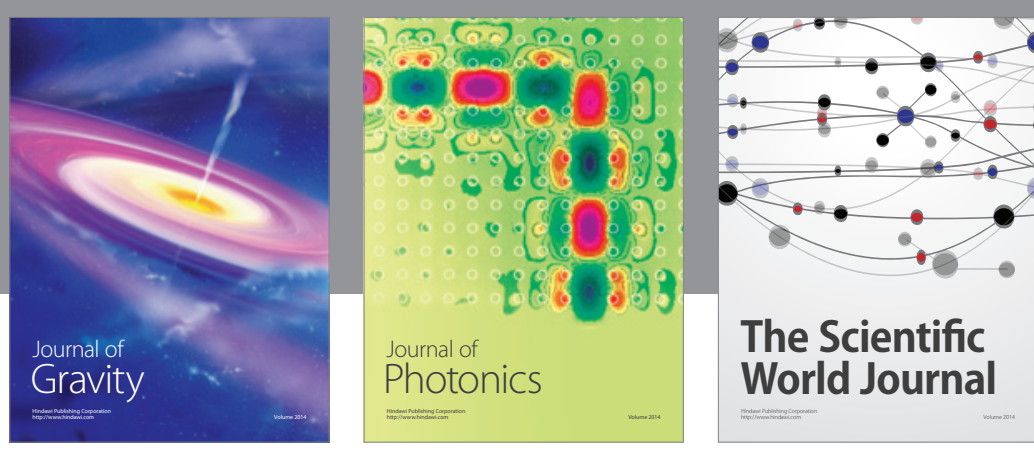

The Scientific World Journal
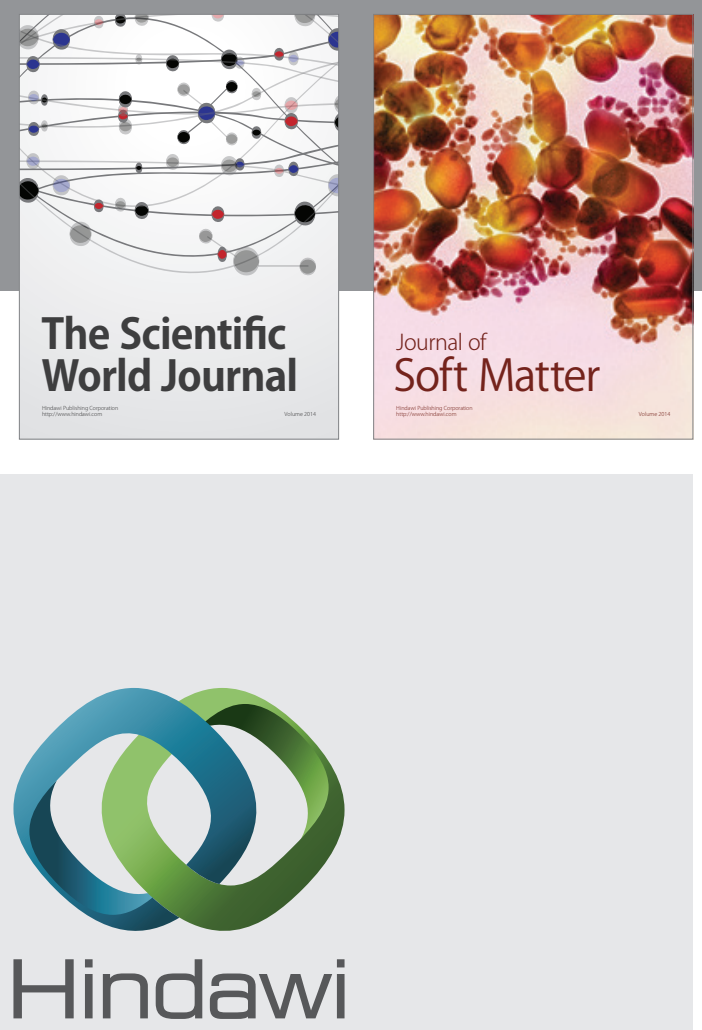

Submit your manuscripts at

http://www.hindawi.com

nternational Journal of

Statistical Mechanics
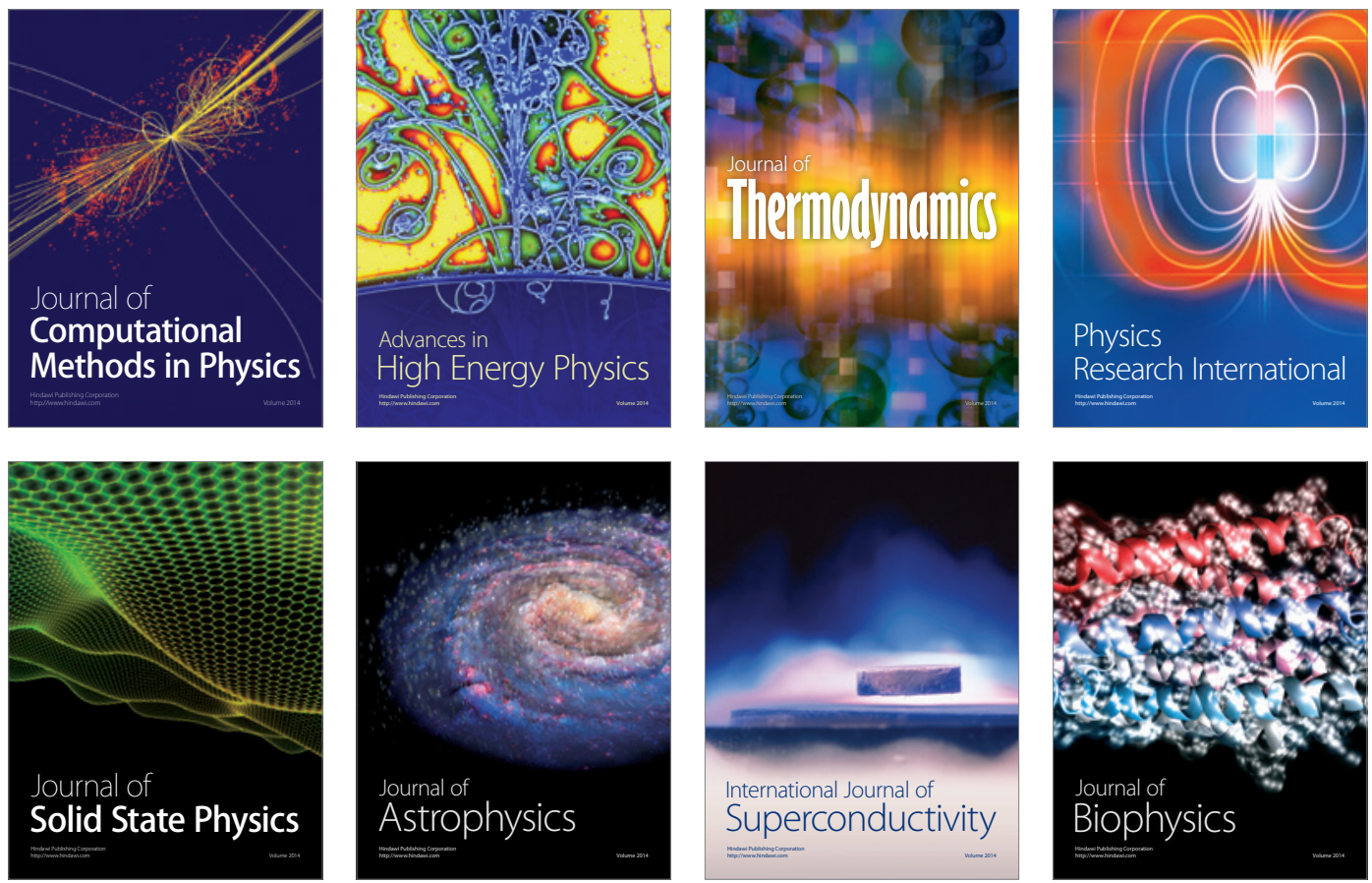
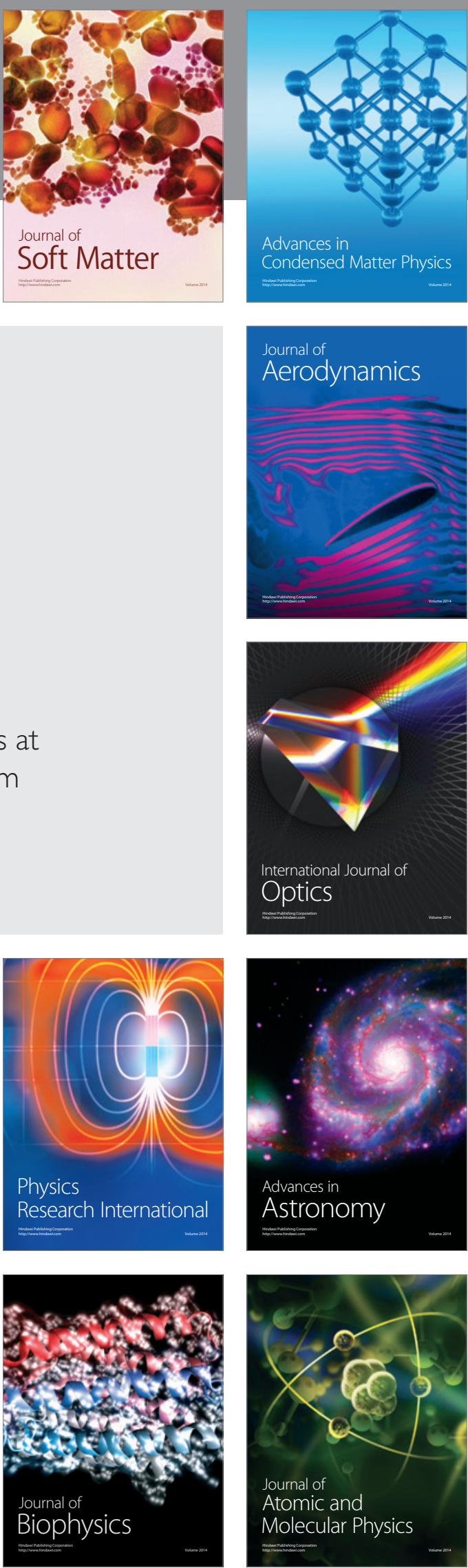